\title{
Interface Design of PC Based on Serial Communication
}

\author{
Yang Yong', Zhao $\mathrm{Li}^{2}$ \\ Tianjin Key Laboratory of Information Sensing and Intelligent Control, Tianjin University of Technology and Education No.1310,Dagu \\ South Road, Hexi District, Tianjin 300222, China
}

\begin{abstract}
At present, the automation equipment is mainly through the serial port to communicate. Mainly through the PC machine as the host computer to carry out the serial port debugging, in the serial communication debugging. Because of the large size of PC and the limitation of industrial field environment, the communication failure cannot be quickly located and eliminated, which increases the difficulty of debugging. By using cross platform $C++$ application development framework $Q T$ to develop portable equipment serial communication interface program, will be portable serial communication interface program is transplanted to the arm development board, through the arm board to serial communication automation equipment debugging, reduce debugging difficulty and improve the working efficiency.
\end{abstract}

Keywords: serial communication, debugging, QT

\section{Introduction}

With the development of science and technology, the degree of industrialization of the factory is getting more and more, and the status of automation technology and equipment in the industrial production is becoming more and more important. In the automation equipment installation, commissioning and maintenance in the process of communication between devices is indispensable. Serial communication is a computer communication mode is used widely, most computer contains two serial interface based on RS232 [1-2]. Data acquisition device inside the plant, mostly rely on serial communication to achieve. Automation equipment debugging mainly through the PC machine, but due to the complexity of the industrial production environment, the use of PC machine for automatic equipment communication debugging, and the operation is relatively large. This paper mainly through the QT communication debugging interface to the development of portable devices, the host computer through the ARM board to replace $\mathrm{PC}$ as the automation equipment for serial communication, PC overcome the disadvantage of large volume.

\section{Serial Communication Interface Design based on QT}

\subsection{Programming environment}

QT is a cross platform $\mathrm{C}++$ graphical user application framework; it's signal and slot mechanism, so that objects do not know each other in the case of information, cooperation. QT has excellent cross platform characteristics, object-oriented, rich API, a large number of developments documents and so on [3].

QT the use of a wide range of environment, and the preparation of the program is also very strong portability. Windows operating system is widely used. In the windows system, not because the operating system and increase the difficulty of program development, users can easily use the QT interface software development program.

\subsection{Serial Communication Protocol}

Serial communication, serial by bit send data and receive data byte. You can use a serial communication line to send data at the same time, another line to receive data. It is very simple and can achieve long-distance communication.

The most important parameters: Baud rates, data bits, stop bits and parity bits. The baud rate is the parameter to measure the communication speed; it said the number of transmitted per second bit. The communication baud rate is not the same, will cause the receiving data garbled. Data bit is a parameter to measure the actual data bits in the communication. The computer sends a packet of information, the actual data is not 8 , and the standard is 5,7 and 8 . The packet contains a start / stop bit, data bits and the parity bit. Stop bit represents the last bit of a single packet. Stop not only indicates the transmission end and provides opportunities for computer clock synchronization correction. The data is sent from time to time in the transmission line, and each device has its own clock. There may be no synchronous communication; stop bits can correct their clock synchronization. Parity is a way of serial communication in error detection. The parity bit can determine whether the noise of communication or transmission and reception of data is not synchronized.

\section{3 serial communication property settings}

A key step in the serial programming is set properties of serial communication, the most common attributes set including port, baud rate, data bits, parity bit, stop bits, the data stream etc. In QT, the constructor contains these parameters.

In this structure which provides serial communication baud rate 9600 , data bits is 8 bit, no parity bit and stop bit 1 bit, data flow control, 500ms delay. Struct PortSettingsmyComSetting $=\quad\{$ BAUD9600, DATA_8, PAR_NONE, STOP_1, FLOW_OFF, 500\}; 


\section{International Journal of Science and Research (IJSR) \\ ISSN (Online): 2319-7064}

Index Copernicus Value (2013): 6.14 | Impact Factor (2014): 5.611

Define the communication port number COM1, serial port driver for event driven.

myCom=newWin_QextSerialPort(“com1”,myComSetting, QextSerialBase::EventDriven);

\section{4 serial port related system functions}

Using QT serial communication programming, the main use of 4 system functions: open (), close (), read (), and write ():

\section{Serial port open function}

myCom ->open (QIODevice::ReadWrite);

This function is used to open the serial port, and then the data can be sent and received.

\section{Serial port close function}

myCom->close ();

This function is used to close the serial port; serial port is closed, not in exchange for data. Only belong to the serial port closed, we can set the serial port parameters.

\section{Serial read function}

MainWindow::readMyCom ();

This function is used to read slave data sent to the host computer and saves the data in the form of an array.

\section{Serial sent function}

myCom-> write (ui->sendMsgLineEdit->text ().toLatin1 ()); The function is used to write the serial port, through the function to send control commands.

\section{Communication interface design}

Qt programming interface program has two ways: the traditional way of hand written code and directly in the Designer Qt interface design. The preparation of the interface is similar to our commonly used VB. Interface driver is based on the QT signal and slot mechanism to write, the interface of each component has its own signal and slot function. There are two kinds of relation between signal and slot function, and it is generally connect () connection function to realize the connection of signal and slot. But in QT designer to design the interface, you can refer directly to the QT controls. These controls have their own slot function, in this case the signals and slots need not passed through the function to connect related signals and slots. QT will self-associate of these controls signals and slots.

\subsection{Form Construction}

QT contains a number of different occasions to meet the window parts, the use of these widgets are simple, flexible, can meet the requirements of some special sub category. Will be a large number of QT window components can be combined to create a relatively simple user interface. QT window parts cannot be simply directly divided into control or container, the window parts are both the control is a container, the two have no absolute limits. The use of window parts is more flexible, in order to meet the specific needs of users, it is easy to quilt [4].

\subsection{Interface layout}

The construction form in the QT Designer, select the interface required window controls. To manage the controls placed using QT layout manager with the layout manager used mainly include: QHBoxLayout, QVBoxLayout and QGridLayout. QHBoxLayout horizontal layout manager for the control of the level of display; QVBoxLayout vertical layout manager is responsible for the control of the vertical placement; QGridLayout grid layout manager in the form of grid control. Program interface, using alternating the layout manager can complete most of the interface layout problems, problems with more complex layout, can use their base class QLayout, and define your own layout manager [5]. The dial area through the grid layout manager on the dial button management, parameter setting area through horizontal layout and vertical layout manager on the digital display control management. Reasonable use of layout managers can reduce the tedious work of the layout, speed up the development efficiency of the interface program. The design of communication interface is shown in Figure 1.

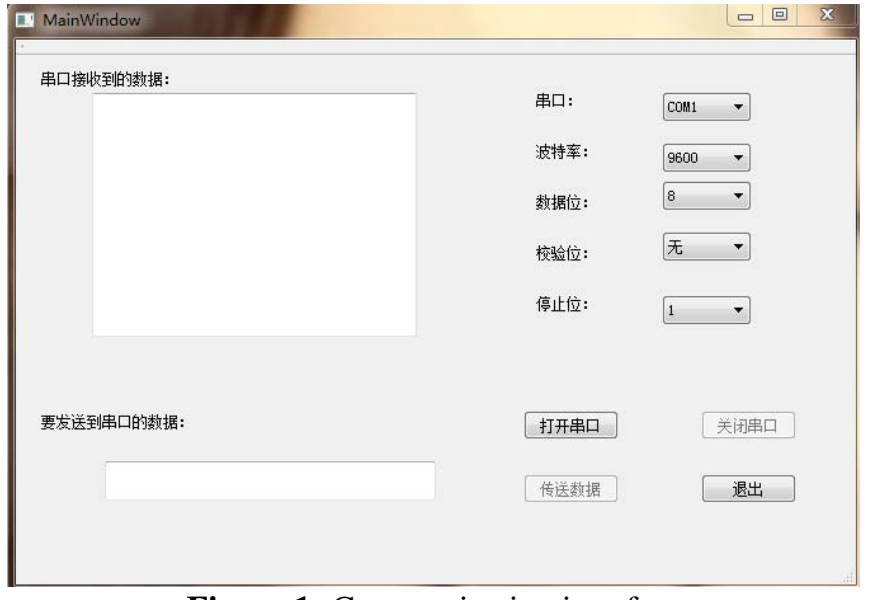

Figure 1: Communication interface

\section{Communication Interface Test}

Communication interface program verification test including the interface program and serial assistant communication and interface program with the microcontroller communication. Through the virtual serial port software to carry out the program verification test, excluding the interference of the hardware circuit to increase the efficiency of development.

\subsection{Communication Interface Debugging}

After compiled serial communication program in QT, execute serial program, serial communication interface will pop up. QT and serial communication assistant without hardware connection, but the communication of the two, need to be connected between the serial ports. Virtual serial port software (VSPD) [6-7], the establishment of a virtual serial port. There is a connection between the virtual serial ports, eliminating the need for hardware connection.

Open the virtual serial port software will pop up in Figure 1 the third dialog box. Virtual serial ports are paired to add, experiment, COM1 and COM2. Each port has a certain 


\section{International Journal of Science and Research (IJSR) \\ ISSN (Online): 2319-7064 \\ Index Copernicus Value (2013): 6.14 | Impact Factor (2014): 5.611}

property, such as port switch, sending and receiving byte number, baud rate etc.

Open the serial port debugging assistant and QT inside the serial procedures, set the serial communication parameters. Through the virtual serial port can see the configuration of the respective serial port property. As shown in Figure 2, the serial assistant to the QT program to send 18765161267 , then QT to the serial assistant to return "nihao" information.

Through the above experimental debugging, successfully realized the serial port assistant and QT communication. Verify the correctness of the serial communication program in QT. The guarantee of correct communication is provided for the next experiment.

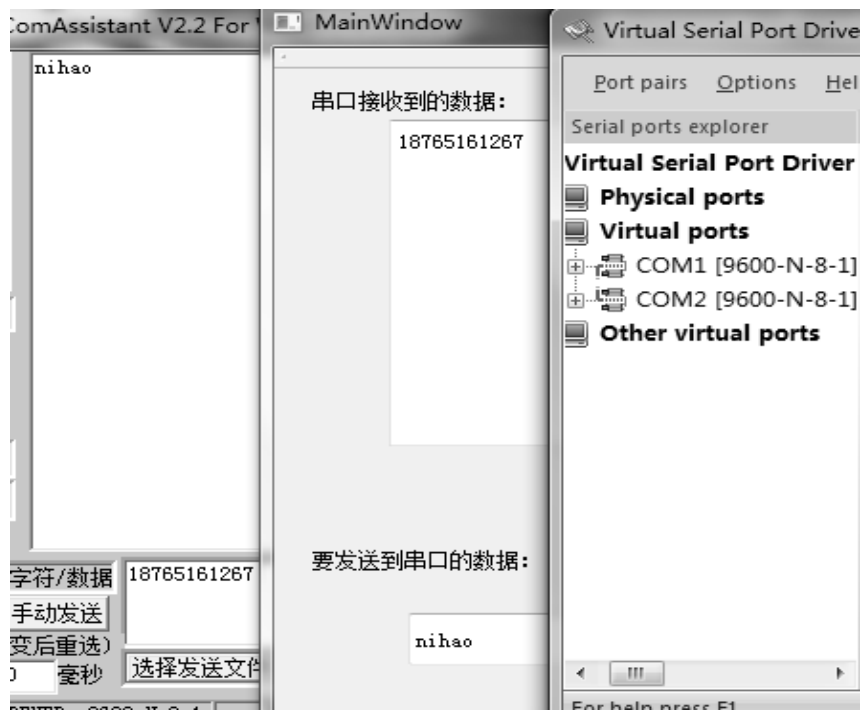

Figure 2: Communication interface

\subsection{QT and MCU communication}

The communication between QT and MCU can also be connected by a virtual serial port. Single chip and serial port need to be bound by the instruction. Or the use of COM1 and COM2, COM1 and QT connection, SCM and COM2 connection. In order to realize the operation of pure software, using keil software instead of single chip microcomputer. Enter debug mode in keil, then the command line at the bottom of the inside, through binding instructions [8]:

\section{MODE COM2 9600,0,8,1}

The instruction set of the 2 serial baud rate, parity bit, data bits, and stop bits. The serial port parameters set in the instruction should be consistent with the serial port parameters in the MCU program.

\section{ASSIGN COM $2<$ SIN $>$ SOUT}

The command completed the microcontroller serial port and COM3 binding. Because the experimental selection of SCM is AT89S52, there is only one serial port, so use SOUT, SIN, if the microcontroller has multiple serial port, you can choose S0IN, S0OUT or S1IN, S1OUT.

Through the above operation to complete the Keil and the serial port 2 binding, the implementation of QT inside the serial program to complete the QT and serial port 1 binding. Keil inside the implementation of the program, the simulation of the temperature data through the serial port to send to the QT, to achieve the detection of temperature. Due to the experimental debugging from the hardware, so that the experimental results cannot be updated in real time, and constantly show 25.5C, as shown in Figure 3. The experiment shows that keil inside the temperature acquisition program can achieve real-time detection of temperature, and the serial communication program is correct. For the future of the hardware debugging to provide the correct procedures for the protection.

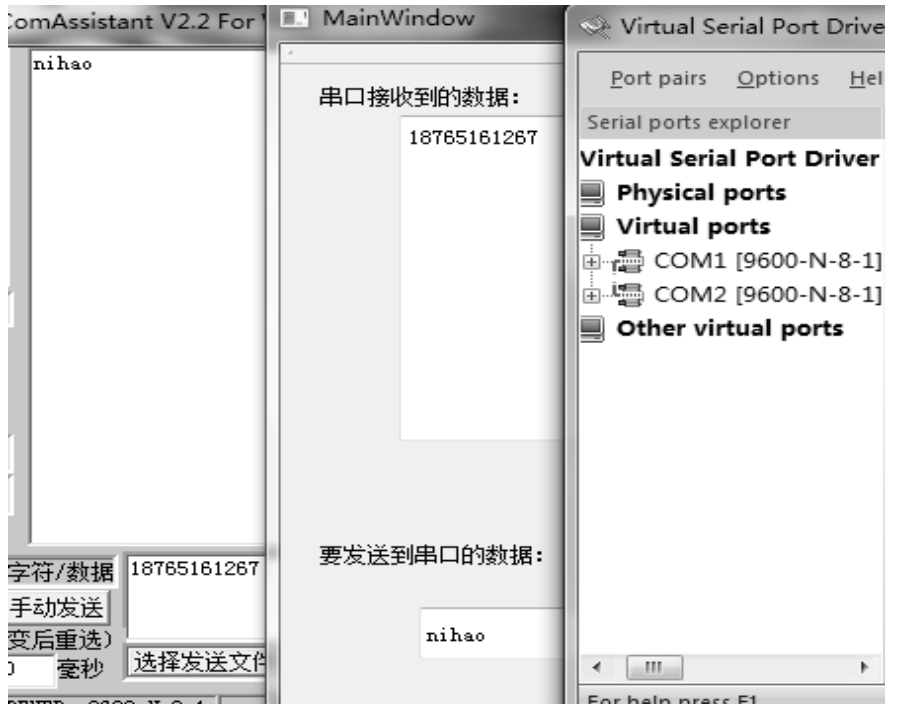

Figure 3: Temperature acquisition

\section{Conclusion}

In learning, usually one to one communication, this kind of communication is relatively easy. The use of serial communication in life is more complex, such as multiple serial ports multiplexing problem, the complexity of the communication data, etc. Excellent communication protocol can greatly improve the accuracy and communication efficiency of serial communication. In this paper, we mainly discuss the method of QT based object oriented programming and the debugging method of pure software which is separated from the hardware. Realized with the interface graphics serial communication applications and pure software serial communication, for the future study of serial communication programming staff to solve the trouble of hardware.

\section{References}

[1] Sun Jianhua, Zhao Xiaodian, GAOYongfei.PC and MCU serial communication system design [J]. Software Guide, 2010, 9 (9).

[2] Wang Jing. Data serial communication based on single chip microcomputer [D]. Yangtze University, 2013.

[3] Di Huihui, Li Jianhua, Liu Jingsang, et al. Design and implementation of the embedded real-time curve display interface based on $[\mathrm{J}]$. Qt/E electronic measurement technology, 2011, 34 (12). 
[4] Yan Xian, Han Xiuling. Research and implementation of serial communication based on [J]. Qt "micro computer information", 2012, 28 (8).

[5] Langley, Nick. "Develop multi-platform interface skills on the Qt,". Computer Weekly. 2007.

[6] http://stormwy.blog.163.com/blog/static/1235293292012 795365591

[7] http://wenku.baidu.com/view/55849f4ffe4733687e21aa99 .html

[8] http://blog.csdn.net/yangluoning/article/details/8035330

\section{Author Profile}

Yang Yong is reading the M.S. degrees in School of Automation from Tianjin University of Technology and Education. 